\title{
Anomalous strength of membranes with elastic ridges
}

\author{
B. A. DiDonna and T. A. Witten
}

James Franck Institute, University of Chicago, Chicago, IL 60637

We report on a simulational study of the compression and buckling of elastic ridges formed by joining the boundary of a flat sheet to itself. Such ridges store energy anomalously: their resting energy scales as the linear size of the sheet to the $1 / 3$ power. We find that the energy required to buckle such a ridge is a fixed multiple of the resting energy. Thus thin sheets with elastic ridges such as crumpled sheets are qualitatively stronger than smoothly bent sheets.

PACS numbers: 68.60.Bs,62.20.Dc,46.25.-y

\section{INTRODUCTION AND THEORY}

When the boundaries of an elastic sheet are sufficiently distorted, singular deformations in the sheet often occur [1, 2, 3, 4, 目, 6]. These singularities are immediately apparent when one crumples a sheet of paper between the hands. Analogous singularities occur whenever the sheet is forced to have points of high curvature [4, 5, 6]. One can readily form such a "puckered" sheet by fastening the edges of that sheet to form disclinations as shown in the cube of Fig. 1. Recently it has been recognized that such structures typically have their energy concentrated into so-called stretching ridges, in which strain and curvature energies are in balance $4,5,6$. The ridge increases the stored elastic energy in the structure dramatically relative to that of a uniformly curved sheet occupying the same region. This enhancement of energy grows as a power of the linear size of the structures relative to the thickness of the sheet.

The large energy content of these structures suggests that they should be strong. They should resist deformation and buckling under external loads qualitatively better than nonsingular structures. In this note we demonstrate this strength by showing that the scaling laws governing the ridge energy also predict its response to loads and its buckling threshold.

We simulate the compression of an elastic cubic shell by the application of external, inward-pointing forces at its vertices. The cube is formed from a flat sheet as shown in Fig. 1. With this connectivity the sheet naturally forms stretching ridges along the edges of the cube. We push on the vertices of the cube until the ridges buckle into several smaller ridges. By rescaling the compressed configuration with the same thickness scaling laws as those appropriate for the resting configuration, we demonstrate that the response of the ridge to this kind of forcing is completely described by the $1 / 3$ power ridge scaling solution determined in [4]. We also demonstrate that the onset of the buckling instability follows this scaling as well.

Ridge scaling comes about as a way to balance bend- ing and stretching energy costs. It has been shown [4] that the important dimesionless parameter governing the elastic energy of a single ridge of length $X$ and material thickness $h$ is the aspect ratio

$$
\lambda=\frac{\sqrt{\kappa / Y}}{X}=\frac{1}{\sqrt{12\left(1-\nu^{2}\right)}}\left(\frac{h}{X}\right),
$$

where $Y$ is the 2-dimensional Young's modulus of the material, $\kappa$ is an effective bending modulus, and $\nu$ is its Poisson ratio. If all lengths are expressed in units of $X$ and all energies in units of $\kappa$, the equilibrium configurations of a thin sheet are completely described by a dimensionless form of the von Kármán equations,

$$
\nabla^{4} f=[\chi, f]+P, \lambda^{2} \nabla^{4} \chi=-\frac{1}{2}[f, f],
$$

where all derivatives are taken with respect to the dimensionless variables $x / X$ and $y / X$. Here the bracket product represents

$$
[a, b]=\epsilon_{\alpha \mu} \epsilon_{\beta \nu}\left(\partial_{\alpha} \partial_{\beta} a\right)\left(\partial_{\mu} \partial_{\nu} b\right),
$$

$P$ is an arbitrary external pressure field acting normal to the surface (in units of $\kappa X^{3}$ ), and the potential fields are related to the curvature $C_{\alpha \beta}$ and stress $\sigma_{\alpha \beta}$ by

$$
\begin{gathered}
C_{\alpha \beta}=X^{-1} \partial_{\alpha} \partial_{\beta} f \\
\sigma_{\alpha \beta}=\kappa X^{-2} \epsilon_{\alpha \mu} \epsilon_{\beta \nu} \partial_{\mu} \partial_{\nu} \chi .
\end{gathered}
$$

This form of $\sigma_{\alpha \beta}$ automatically satisfies the equilibrium condition $\partial_{\alpha} \sigma_{\alpha \beta}=0$.

Since $\lambda$ comes into the von Kármán equations multiplying the stess source term, the possible configurations of a thin elastic sheet are well described by a stress free, $\lambda=0$ folding solution plus boundary layers at the fold lines. Lobkovsky's insight in $⿴$ was to try a scaling solution for the boundary layer of a single ridge which matched the $f$ scaling of the outer, sharp fold solution. For a fold of dihedral angle $\alpha$ across the line $y=0, f=\alpha|y / X|$. Accordingly, on the boundary layer $f$ should scale with 
the same power of $\lambda$ as the dimensionless transverse coordinate $y / X$. Substituting a scaling form into the dimensionless von Kármán equations with $P=0$ and equating the highest order terms yields scaling of the form

$$
f=\lambda^{1 / 3} \tilde{f}, \chi=\lambda^{-2 / 3} \tilde{\chi}, y=\lambda^{1 / 3} X \tilde{y}, x=X \tilde{x},
$$

where the tildes denote dimensionless, scale free coordinates and functions. This translates to $\lambda^{1 / 3}$ scaling of the boundary layer width, $\lambda^{-1 / 3}$ scaling of the transverse ridge curvature, and $\lambda^{2 / 3}$ scaling of the strain along the ridge length.

External forcing applied to the sheet enters the von Kármán equations via the term $P$ and via boundary conditions at the sheet's edges. In this research we consider an external potential which essentially applies point forces to either end of a ridge. Since the spatial extent of the applied force is a delta function we do not expect it to destroy the spatial scaling of the ridge solution. Therefore we may reasonably expect to find that the equilibrium configuration of a ridge under a given compressive force is identical to rescaled configurations of ridges with different material thickness and properly rescaled external force magnitudes.

To calculate the proper rescaling of the forces on the vertices for a similarity solution, we consider our forcing as a boundary condition consisting mainly of an in-plane point force. This force amounts to a point stress at the edge of the sheet with the form

$$
\sigma_{x x}^{(o)}=F_{o} \delta(y) .
$$

So, to find similar scaled configurations of the sheet, we must scale $\sigma_{x x}^{(o)}$ the same way $\sigma_{x x}$ scales on the ridge. Since $\gamma_{x x}$ scales as $\lambda^{2 / 3}$, and

$$
\sigma_{x x} \sim Y \gamma_{x x}=\kappa(\lambda X)^{-2} \gamma_{x x},
$$

then $\sigma_{x x} \sim \kappa \lambda^{-4 / 3} X^{-2} \tilde{\gamma}_{x x}$, where $\tilde{\gamma}_{x x}$ is a dimensionless, scale free function. To express $\sigma_{x x}^{(o)}$ in similar fashion, we substitute the scale free $y$-variable $\tilde{y}=\lambda^{-1 / 3} y / X$, so that $\delta(y)=\lambda^{-1 / 3} X^{-1} \delta(\tilde{y})$. Thus, the proper scale free force can be written in terms of $F_{o}$ as

$$
F_{o}=\frac{\kappa \lambda^{-1}}{X} \tilde{F}_{o} .
$$

For reasons which will become clear in the next section, we cannot measure the force we apply to our ridge with very good accuracy. However, we can measure the inward displacement $\Delta$ of the ridge ends caused by this forcing. These two quantities may be related by assuming that the work done by equivalent rescaled forces, given approximately by $F_{o} \Delta$, scales the same as the total energy of the resting ridge configuration. The total energy of a resting ridge scales as $\kappa \lambda^{-1 / 3}$, so equivalent values of $F_{o} \Delta / \kappa$ will scale as $\lambda^{-1 / 3}$. Given the scaling of $\tilde{F}_{o}$ from Eq.8, the scale free $\tilde{\Delta}$ must be related to the actual displacement by

$$
\Delta=\lambda^{2 / 3} X \tilde{\Delta}
$$

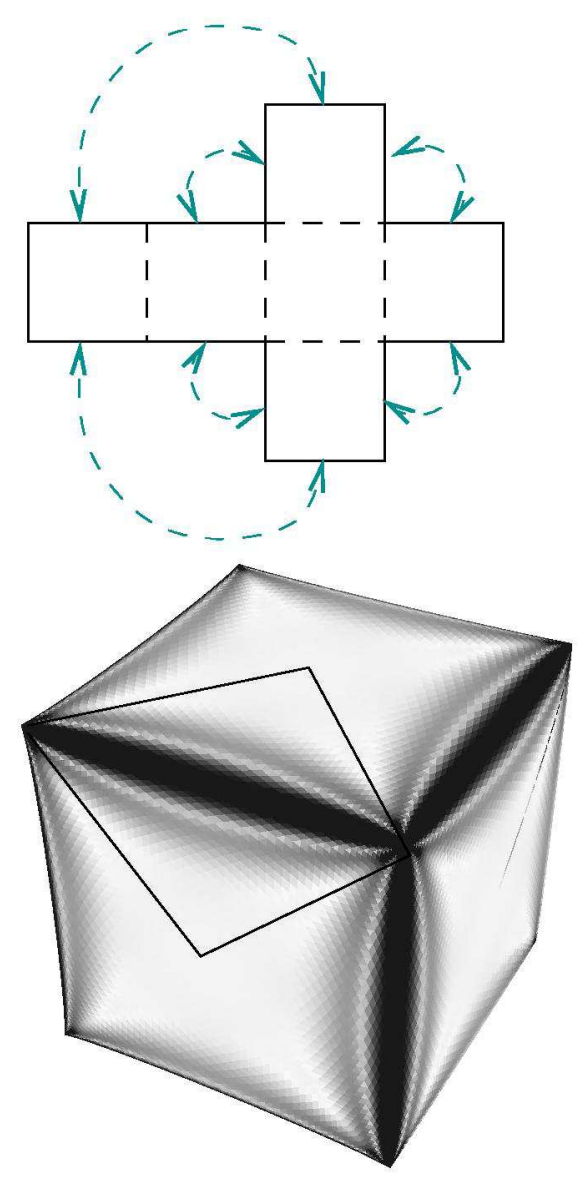

FIG. 1: Typical elastic sheet used in this study. The top image illustrates how a flat sheet is joined at its edges to form a cube. The bottom image shows the resting configuration of the cube with no external forces acting when the thickness of the sheet is .0004 of the the edge length and the Poisson ratio is $1 / 3$. Darker shading represents higher strain energy density. The energy was minimized in the outlined diamondshaped region; the rest of the cube shape was inferred by symmetry. Slight numerical symmetry breaking between the left and right sides of the diamond created slight mismatches of the inferred surfaces on other faces, such as the right-hand face. The numerical grid is visible as a quiltlike texture. It has a finer scale at the edges and corners where curvature is larger.

\section{NUMERICS}

To test these scaling predictions we simulate a flat elastic sheet joined to form a cube as shown in Fig. 1. The sheet is a triangular grid with variable grid spacing. Strains and curvature are taken to be constant across the face of each triangle, with curvature calculated relative to the local normal at each triangle. Bending and stretching energies were assigned to the curvature and strains on each triangle using the forms for elastic energy presented in [7] for a sheet of elastic thickness $h$ and Poisson ratio 


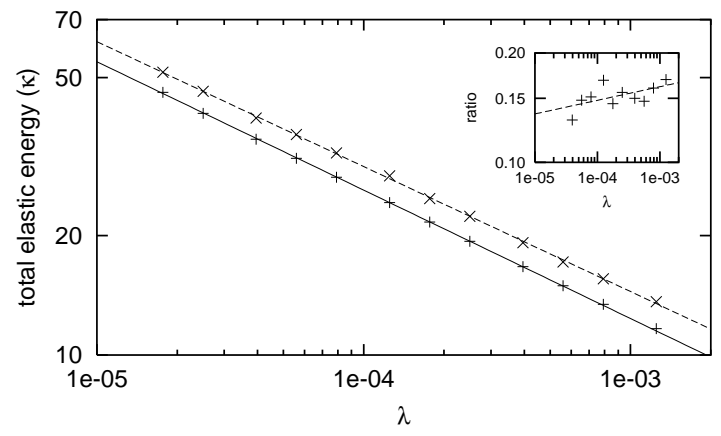

FIG. 2: Energy of ridges at rest and at the buckling threshold. Straight lines are least squares fits to a scaling form $y=a x^{b}$. In this plot $\lambda$ ranges from $1.25 \times 10^{-3}$ to $1.77 \times 10^{-5}$. The plot shows the total elastic energy $\left(E_{B}+E_{S}\right)$ in the sheet after minimization. The scaling exponent fit for the resting ridge values (lower line) was -0.32 , the fit at the buckling threshold was -0.31 . The inset shows the difference between threshold energy and resting energy in units of the resting energy. This energy ratio is best fit by a scaling exponent of $0.05 \pm 0.02$ and is consistent with a constant ratio.

$1 / 3$. The gridding was chosen to have smooth gradients in triangle density over most of the surface while concentrating the lattice spacing at the vertices by a factor of $10^{3}$ and across the ridge-line by a factor of $10^{2}$ compared to the flat regions far from the ridge. The concentrations factors were chosen arbitrarily, within the limits of the mapping, to make the gridding near the vertices as fine as possible.

Pushing on the tips of the cube is accomplished by introducing repulsive potentials of the form $V(r)=$ $C_{p} /\left|\vec{r}-\vec{x}_{p}\right|^{2}$ centered around points $\vec{x}_{p}$ which lie just outside the vertices of the cube. To prevent rotation, the vertices are explicitly constrained to lie on radial lines that pass through the center of the cube and the points $( \pm 1, \pm 1, \pm 1)$ which define the vertices of a perfect cube. The center points of the pushing potentials are located on these radial lines, at the point where the vertex would lie if the sheet were sharply folded at the ridges - relaxation of the ridge curvature draws the vertices inward from these points for an unforced resting ridge. This potential concentrates the forces at the corners without creating lattice-scale numerical instabilities. $C_{p}$ was varied to apply different loading.

An inverse gradient routine [9] was used to minimize the total elastic and potential energy of the sheet as a function of the coordinates of all the lattice points for given parameters $\kappa, Y$ and $C_{p}$.

To save computational time, energy was computed and minimized on only one ridge-line (the diamond shaped region highlighted in Fig. 11). The positions of points on the rest of the cube were calculated by reflection across symmetry planes. This constraint explicitly required that the edges of the simulated region be confined to the sym- metry reflection planes. Thus many forms of deformation that break the symmetry of the resting state are not possible in the simulation. However, the minimization may break the left-right symmetry of the diamond-shaped region, and we found that it does so to a slight degree. The assymmetry shows up in our reconstruction as a slight mismatch in other faces of the cube, as noted in Fig. 1. We do not believe these mismatches are important for the scaling phenomena we report.

We found minimum energy configurations for ridges of aspect ratio $\lambda$ ranging from $1.25 \times 10^{-3}$ to $1.77 \times 10^{-5}$. The upper bound on $\lambda$ was determined by the range of validity of the ridge scaling solution - above this value the width of the ridge becomes comparable to that the sheet. At the other extreme, for $\lambda<10^{-5}$ the radius of curvature at the ridge line becomes comparable to the spacing of our lattice and the simulation ceases to be accurate. For each value of $\lambda$ we first found the minimum energy configuration with no applied forces. This configuration was then used as the initial condition to find minimum total energy configurations for the ridge in the presence of the tip-pushing potential described in the numerics section. We made the tip-forcing progressively stronger by increasing the potential coefficient $C_{p}$ in constant steps, each time evolving the grid to an energy minimizing configuration. The forcing was increased until the ridge buckled. Buckling of the ridge was marked by the appearance of new points of sharp curvature along the ridge as well as a sudden decrease in the total elastic energy $E_{S}+E_{B}$ of the sheet. Thus the unbuckled sheet is metastable. We made no attempt to scale the size of the potential step with the thickness of the sheet, so while it took nearly 30 steps to buckle the ridge in thicker sheets, it only took 6 or 8 steps for the thinnest sheets.

\section{FINDINGS}

The plot in Fig. 2 shows scaling of the total elastic energy in the cube versus $\lambda$ for ridges at rest $\left(C_{p}=0\right)$ and at the buckling threshold. Scaling of the total elastic energy for the resting configuration is consistent with a $\kappa \lambda^{-1 / 3}$ dependence, in agreement with prior theory and simulation [4, 6]. Fig. 2 2 also shows that the elastic energy measured at the buckling threshhold exhibits exactly the same scaling as on resting ridges. This suggests that the response of the cube to our tip-forcing is completely determined by the ridge, and that the particular form of our forcing potential does not destroy the length scaling of the ridge. The inset in Fig. 2 shows that that the energy correction at the buckling threshold is nearly a constant fraction of the total ridge elastic energy.

Scaling of the force response is verified by the demonstration presented in Fig. 3 of a similarity solution for ridge shape. Plot (a) in this figure shows values of $C_{y y}$, along a line in the material coordinates which bisects the 

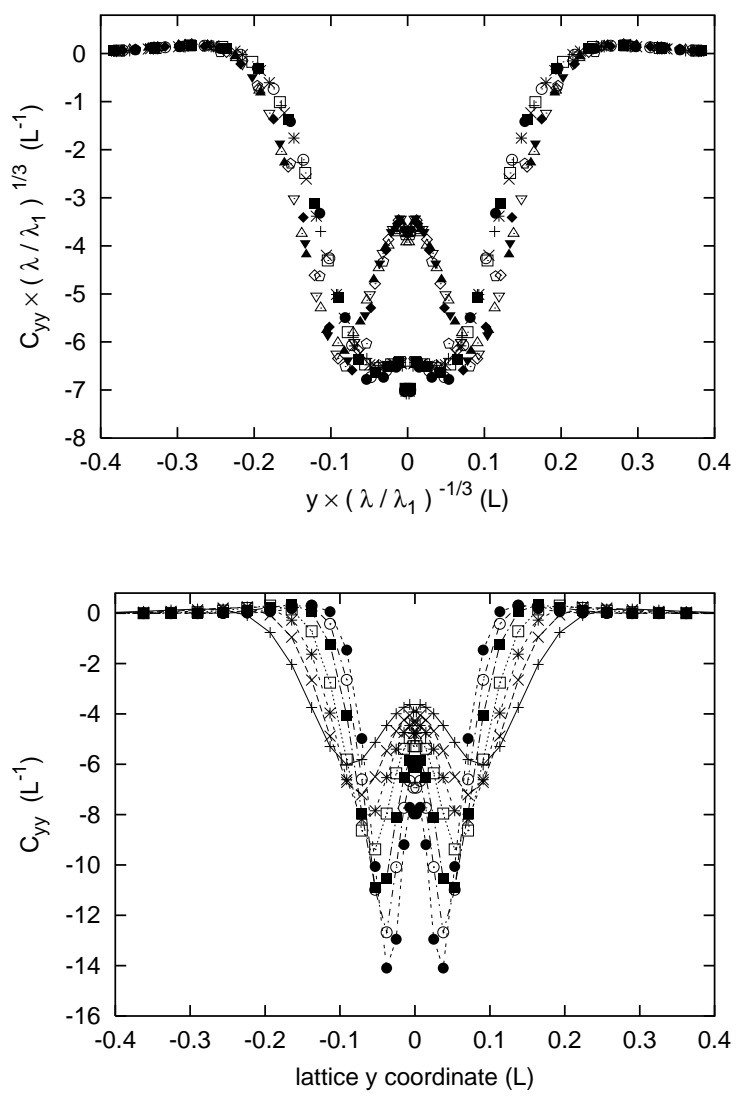

FIG. 3: Demonstration of a similarity solution for the ridge response to forcing. Both plots show $C_{y y}$, the curvature across the ridge-line, versus the $y$ material coordinate on the line which bisects the ridge-line. The data is for sheets with seven different values of $\lambda$, ranging from $1.25 \times 10^{-3}$ to $1.25 \times 10^{-4}$. Plot (a) shows $C_{y y} \times\left(\lambda / \lambda_{1}\right)^{1 / 3}$ vs. $y \times\left(\lambda / \lambda_{1}\right)^{-1 / 3}$ for the ridges at rest and for ridges with inward vertex displacement $\Delta(\lambda)=\Delta_{1}^{(c)} \times\left(\lambda / \lambda_{1}\right)^{0.67}$, where $\Delta$ is measured from the resting vertex positions, $\lambda_{1}$ is the aspect ratio for the thickest sheet, and $\Delta_{1}^{(c)}$ was vertex displacement at the buckling threshold for the thickest sheet. The profiles with the large central peak are the buckling threshold values. (The small dimple in the data at $y=0$ is a numerical artifact due to a discontinuity in the gridding density across the ridge-line. For finer gridding this dimple goes away, while all other local values of curvature remain constant.) Plot (b) shows unscaled $C_{y y}$ versus $y$ for the buckling threshold profiles plotted in (a).

simulated ridge line, for several different sheet thicknesses and two different values of rescaled ridge tip displacement $\Delta$. We tested values of $\Delta$ which spanned the ridge re- sponse from zero forcing to the point of buckling. The magnitude of $C_{y y}$ was rescaled by $\left(\lambda / \lambda_{1}\right)^{1 / 3}$ and the $y$ coordinate was rescaled by $\left(\lambda / \lambda_{1}\right)^{-1 / 3}$. We found that the rescaled $C_{y y}$ profiles mapped onto each other best if the values of $\Delta$ used for each sheet thickness were determine by rescaling $\Delta_{1}$, the value used for the thickest sheet, by $\left(\lambda / \lambda_{1}\right)^{0.67}$, where $\lambda_{1}$ is the aspect ratio of the thickest sheet. For comparison, the unscaled $C_{y y}$ versus $y$ for a particular rescaled $\Delta$ is shown in Fig. 3(b). The rescaling exponents used for $C_{y y}$ and $y$ were taken from the ridge scaling solution. The $\Delta$ rescaling exponent is very close to the theoretical value of $2 / 3$ derived above.

We have demonstrated scaling of the ridge response to a typical form of external forcing. Since the buckling threshold of the ridge under external forcing is determined by the energy scaling of the resting ridge solution, the relatively high elastic energy content of very thin ridges translates directly into increased ridge strength. We are actively pursuing a better understanding of the buckling properties of this distinctive elastic structure.

This work was supported in part by the National Science Foundation under Award number DMR-9975533.

[1] M BenAmar, Y Pomeau, "Crumpled Paper" P Roy Soc Lond A Mat 453, 729 (1997).

[2] E. Cerda, S. Chaïeb, F. Melo, L. Mahadevan, "Conical Dislocations in Crumpling" Nature 401, 46 (1999).

[3] A. Boudaoud, P. Patricio, Y. Couder, M. Ben Amar, "Dynamics of singularities in a constrained elastic plate" Nature 407, 718 (2000).

[4] A. E. Lobkovsky, "Boundary Layer Analysis of the Ridge Singularity in a Thin Plate" Phys Rev E 53, 3750 (1996).

[5] A. E. Lobkovsky, T. A. Witten, "Properties of Ridges in Elastic Membranes" Phys Rev E 55, 1577 (1997).

[6] A. Lobkovsky, S. Gentes, H. Li, D. Morse, T. A. Witten, "Scaling Properties of Stretching Ridges in a Crumpled Elastic Sheet" Science 270, 1482 (1995).

[7] H. S. Seung and D. R. Nelson, "Defects in Flexible Membranes with Crystalline Order" Phys Rev A 38, 1005 (1988).

[8] L. D. Landau and E. M. Lifshitz, Theory of Elasticity (Pergamon Press, New York, 1959).

[9] W. H. Press, S. A. Teukolsky, W. T. Vetterling and B. P. Flannery, Numerical Recipes in $C$ (Cambridge University Press, Cambridge, 1992). 